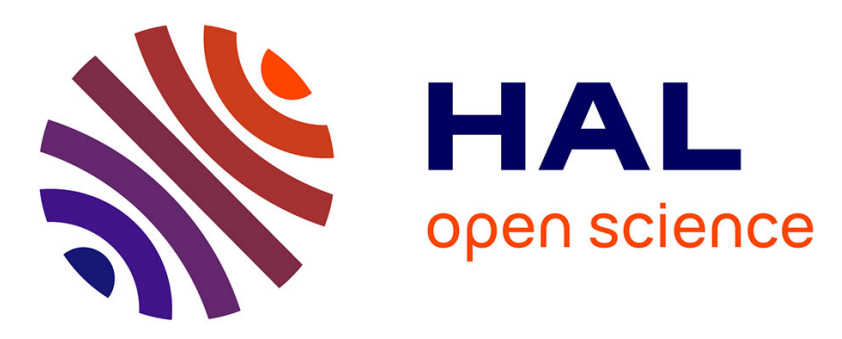

\title{
A Three-Tiered Architecture for Large-Scale Wireless Hospital Sensor Networks
}

Jamila Ben Sliman, Ye-Qiong Song, Anis Koubâa, Mounir Frikha

\section{To cite this version:}

Jamila Ben Sliman, Ye-Qiong Song, Anis Koubâa, Mounir Frikha. A Three-Tiered Architecture for Large-Scale Wireless Hospital Sensor Networks. Workshop MobiHealthInf 2009 in conjunction with BIOSTEC 2009, Jan 2009, Porto, Portugal. pp.64. inria-00435508

\section{HAL Id: inria-00435508 https://hal.inria.fr/inria-00435508}

Submitted on 24 Nov 2009

HAL is a multi-disciplinary open access archive for the deposit and dissemination of scientific research documents, whether they are published or not. The documents may come from teaching and research institutions in France or abroad, or from public or private research centers.
L'archive ouverte pluridisciplinaire HAL, est destinée au dépôt et à la diffusion de documents scientifiques de niveau recherche, publiés ou non, émanant des établissements d'enseignement et de recherche français ou étrangers, des laboratoires publics ou privés. 


\title{
A Three-Tiered Architecture for Large-Scale Wireless Hospital Sensor Networks
}

\author{
Jamila Ben Slimane $^{1,2}$ Ye-Qiong Song ${ }^{2}$ Anis Koubâa ${ }^{3,4}$ Mounir Frikha ${ }^{1}$ \\ ${ }^{1}$ Sup'Com, City of Communication Technologies, 2083 Ariana, Tunisia \\ ${ }^{2}$ LORIA-INPL, LORIA -Campus Scientifique, BP 23954506 Vandoeuvre-lès-Nancy, \\ France \\ ${ }^{3}$ IPP-HURRAY! Research Group, Polytechnic Institute of Porto, Rua António Bernardino \\ de Almeida, 431, 4200-072 Porto, Portugal \\ ${ }^{4}$ Al-Imam Muhammad ibn Saud University, Computer Science Dept., \\ 11681 Riyadh, Saudi Arabia \\ jamilabs07@yahoo.fr, song@loria.fr, akoubaa@dei.isep.ipp.pt, \\ m.frikhadsupcom.rnu.tn
}

\begin{abstract}
The Utra Wide Band physical layer specified by the IEEE 802.15.4a standard [1] presents numerous advantages comparing with its original IEEE 802.15.4 standard, namely high accuracy positioning ability, high data rate up to $27 \mathrm{mbps}$, extended communication range, low power consumption and low complexity.

Actually, many research and development activities focus on the design of UWB sensor nodes entities. However nodes interactions or network configuration are neglected. For that, we propose in this paper to investigate the use of UWB for large scale Wireless Hospital Sensor Networks (WHSNs) to benefit from the advantages offered by the UWB technology. This evolving networking paradigm promises to revolutionize healthcare by allowing inexpensive, non-invasive, pervasive and ubiquitous, ambulatory health monitoring. We present the design of new system architecture, based on IEEE 802.15.4a compliant sensors, suitable for health monitoring application in high dense hospital environment. The proposed system architecture is intended to support large-scale deployment and to improve the network performance in terms of energy efficiency, real-time guarantees and Quality-of-Service (QoS).
\end{abstract}

\section{Introduction}

\subsection{Motivation}

Ultra-Wide Band (UWB) technology [2] has recently been quite attractive to the wireless community. Indeed, this emerging technology promises high-rate, low power transmission, immunity to multipath propagation and high-precision ranging capabilities. It represents an ideal candidate technology for many Wireless Sensor Networks (WSNs) application areas such as Wireless Body Sensor Networks (WBSNs).

This recent technological advance in wireless sensor systems offers great potential for the design of low-cost, miniature, lightweight, and intelligent physiological sensor-based applications. These sensor nodes, which are capable of sensing, 
processing, and communicating one or more vital signs, can be seamlessly integrated into wireless personal or body networks for health monitoring. Currently, this technology is being investigated for use in Body Sensor Networks (BSNs) [3-7]. Reference [3] has proposed a design of an UWB transmitter for WBSNs and it mentions that the probable topology for BSNs will be a star network, which can be related to a standard telecommunication infrastructure such as WLAN, cellular networks or fixed telephony network. In addition, the authors in [4] have evaluated the multi-user interference (MUI) effect of the UWB Physical Layer (PHY) proposed by the IEEE 802.15.4a in a star-based Impulse Radio-UWB BSN for medical and sports applications. In [5], the authors have suggested a medical picture transmission service using IEEE 802.15.4a specification, and it has proposed a propagation scheme to solve the problem of interference from the medical equipments simultaneously active in same workspace. In all these previous works [3-5], the authors have been interested in evaluating the IEEE 802.15.4a UWB PHY without considering (1) the impact of higher-layers (Medium Access Control (MAC), network topology, routing policy) and (2) the optional features proposed by the standard that can really enhance $\mathrm{BSN}$ performances.

Contributions of the paper. In this paper, we propose a new Wireless Hospital Sensor Network (WHSN) three-tiered architecture in order to support large-scale deployment and to improve the network performance in terms of energy efficiency, real-time guarantees and QoS. Moreover, we design a simple but efficient solution that optimally allocates channel in large-scale WHSNs, which facilitates mobility and duty cycle management. We are particularly interested in the use of UWB as a key technology for our solution given the extremely wide bandwidth of such signals offering several advantages including high data capacity, low probability of interference, low power consumption, localization capability, low complexity, low cost and the co-existence with other systems.

The paper is organized as follows. Section 2 provides a survey of the UWB physical layer characteristics supported by the IEEE802.15.4a standard. Section 3 presents the proposed system architecture to a hospital dense network.

\subsection{Related Work}

In the context of healthcare and medical applications, the choice of a system model and the definition of the interactions between network members play an important role in the design of WBSNs allowing more accurate monitoring of life critical parameters, enhancement of performance and mobility support. For example, the solution proposed in [6] consists of a two-tiered sensor network using a clustered architecture with a star elementary wireless network. First, we note that the use of the static TDMA scheme with a star topology inside a cluster limits the density of a cluster and then affect the scalability of the network. Moreover, such scheme is less suitable for health monitoring in heterogeneous high dense hospital environment with different states of mobile patients generating continuous and sporadic traffic, for which we should propose adaptive network configuration. References [7-9] propose three-tiered WBSN architectures for home medical supervision. As in [6], authors in 
[8] propose a centralize TDMA medium access protocol that is more suitable for small networks rather than dense networks. Reference [10] proposes a telemedicine system based on ZigBee BSN associated with $3 \mathrm{G}$ networks. However, the UWB physical layer specified by the IEEE 802.15.4a standard offers more important data rates than supported by physical layers of actual Zigbee or Bluetooth devices.

Where only references $[11,12]$ are interested in hospital system design, where authors have proposed flat tree BSNs architecture with three levels for hospital environment based on IEEE 802.15.4 sensors.

\section{Survey of UWB IEEE 802.15.4a Physical Layer}

In addition to the existing physical layer features specified in the original IEEE802.15.4 standard, the recent IEEE 802.15.4a standard [1] offers two new optional physical layers (PHYs): UWB PHY and Chirp Spread Spectrum CSS PHY. The physical layer of the IEEE802.15.4a protocol supports the following operations and parameters:

1. Activation and deactivation of the radio transceiver.

2. Determine Energy Detection (ED) parameters within the current channel: the estimation of the received signal power within the bandwidth.

3. Extract Link Quality Indicator (LQI) for received packets: the characterization of the Strength and/or Quality of a received signal on a link.

4. Perform Clear Channel Assessment (CCA): This mechanism is responsible of reporting the medium activity state: busy or idle.

5. Perform channel frequency selection.

6. Data transmission and reception with several data rates varying approximately between $0.11 \mathrm{Mbps}$ and 27.24 Mbps.

7. Optional feature of precise ranging (UWB PHY).

\subsection{PHY Channels}

According to the IEEE 802.15.4a standard, UWB devices can operate in three independent bands: (1) the sub-gigahertz band (250-750 MHz), (2) the low band $(3.1-5 \mathrm{GHz})$ and (3) the high band $(6-10.6 \mathrm{GHz})$. Fig. 1 gives the center frequencies and bandwidths of the admissible bands, as well as the regulatory domains in which they are admissible.
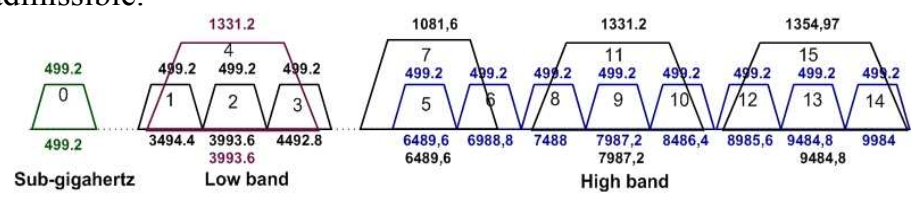

USA : $0-1$

Europe : 1-9

Japan : 2-4 and 8-15

Fig. 1. IEEE 802.15.4a UWB plan bands

The extremely wide bandwidth of UWB signals offers several advantages including high data capacity, low probability of interception and interference, high 
time resolution, low complexity, low power consumption, low cost and the coexistence with other systems.

\subsection{Frame Structures}

Fig. 2.a and Fig. 2.b illustrate the UWB PHY frame with preamble sense based on the Synchronization Header (SHR) of a frame and the UWB PHY frame with preamble sense based on the packet with the multiplexed preamble, respectively. The UWB frame is composed of three major components: the SHR preamble, the PHY Header (PHR), and the Physical layer Service Data Unit (PSDU).

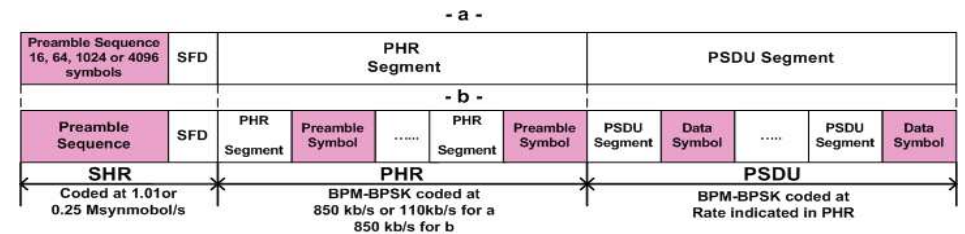

Fig. 2. -a-IEEE 802.15.4a UWB frame structure. -b-IEEE 802.15.4a UWB frame with multiplexed preamble

\subsection{Data Rates}

For UWB PHY, the new standard defines several data rates including $110 \mathrm{kbps}, 850$ kbps, $6.81 \mathrm{Mbps}$ and $27.24 \mathrm{Mbps}$ and a variety of options that give IEEE $802.15 .4 \mathrm{a}$ compliant devices a high degree of flexibility. The data rate depends on the set of PSDU rate-dependent parameters (bandwidth, preamble code length and modulation and coding) and timing-related parameters (number of possible burst positions per symbol and burst duration and symbol duration).In a Personal Area Network (PAN), the network beacon broadcasts must be at the mandatory rate $(850 \mathrm{kbps})$ for synchronization reasons. Devices are allowed to use optional data rates when communicating with each other, these rates are provided to allow devices in close proximity to shorten their transmission duty cycle.

\subsection{Power Consumption}

The highest allowable limits for UWB emission are based on an equivalent emission power spectral density (PSD) of $-41.3 \mathrm{dBm} / \mathrm{MHz}$. A comparative study between the energy consumption magnitude of the IEEE 802.15.4a standard and its original the IEEE 80.215.4standard mentions that for the recent standard transmit powers cannot exceed $37 \mu \mathrm{W}$ and $96.3 \mu \mathrm{W}$ respectively with $500 \mathrm{MHz}$ and $1354 \mathrm{MHz}$ of bandwidth, where the majority of the original standard devices are expected to operate with transmit powers between $0.5 \mathrm{~mW}$ and $10 \mathrm{~mW}$, with $1 \mathrm{~mW}$ being the typical value. Thus, power consumption is obviously much better in IEEE 802.15.4a UWB PHY than IEEE 802.15.4 PHYs. 


\section{The Network Model}

In general, BSNs are wireless networks that support the use of biomedical sensors and are characterized by its (1) very low transmit power to coexist with other medical equipments and provide efficient energy consumption, (2) high data rate to allow applications with high QoS constraints,(3) low cost, low complexity and miniature size to allow real feasibility.

These requirements are extremely hard to satisfy and are not met by known elementary wireless network technologies. In order to satisfy those prominent constraints and to deploy a very dense network supporting a considerable number of BSNs, we propose a three-tiered network to represent the WHSN using UWB sensors. In first and second layers of the network architecture, we have opted for the use of UWB technology as a federating communication protocol to take advantage from its extreme low transmit power minimizing interference and coping with health concerns, high data rate allowing real-time and high data rate applications and location capacity allowing mobility management and patient identification. As for the third tier, we propose the use of $\mathrm{WiFi}$ technology to benefit from its high data rate, large coverage and security aspect.

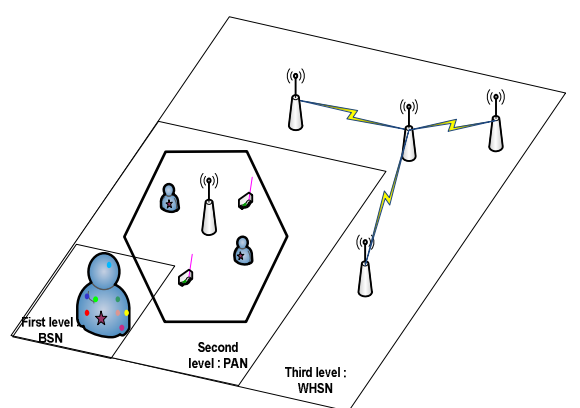

Fig. 3. WHSN architecture "three-tiered cellular network"

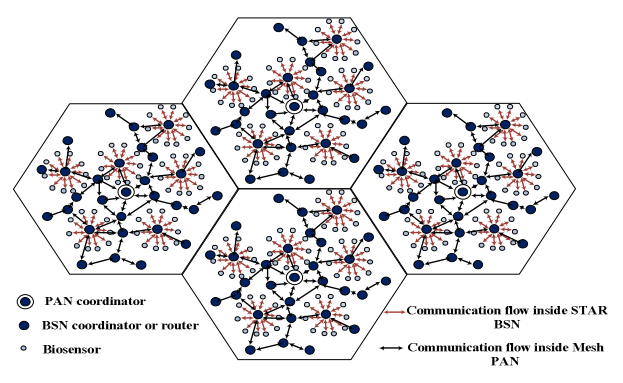

Fig.4. Proposed topologies inside WHSN

Fig. 3 shows all network layers composing the WHSN architecture:

1. First level (or lowest tier) represents the BSN,

2. Second level (or intermediate tier) represents the PAN,

3. Third level (or highest tier) represents the WiFi network.

Fig. 4 shows the proposed topology for each level of WHSN:

1. STAR topology for BSN given the simplicity of such topology,

2. Mesh topology for PAN in order to ensure energy efficiency, real-time guarantees and Quality-of-Service (QoS),

3. Mesh topology for WiFi network in order to ensure real-time guarantees and Quality-of-Service (QoS) in large coverage network. 


\subsection{First Tier: BSN}

The first tier represents the BSN. As shown in the Fig. 5, we represent an elementary BSN by a network with a surface of $2 \mathrm{~m}$ by $2 \mathrm{~m}$ (i.e. $4 \mathrm{~m}^{2}$ ) ensuring the radio coverage of the entire body network. Depending on the state of the patient, approximately dozen of Impulse Radio UWB biosensors including the BSN coordinator can be deployed at the most adequate locations in order to carry out the necessary physiological information for patient health monitoring. Biosensor location, upon the human body, is fixed and is defined according to the type of the biosensor. The $B S N$ coordinator, which is the BSN master node managing all BSN communications, must be located at the center. As compared to its external environment, each BSN is relatively mobile with regards to the others BSNs, routers and its PAN coordinator. In addition, inside one BSN observe a quasi-mobility for biosensors located on the hands, arms and feet.
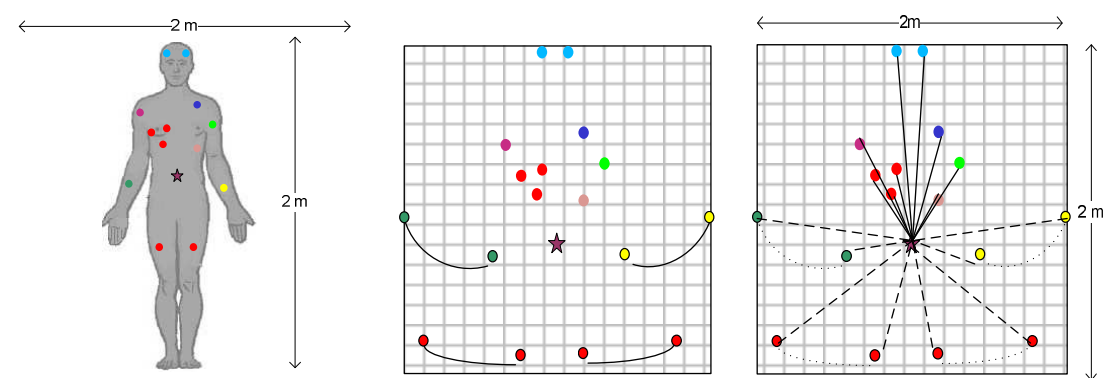

Fig.5. Body Sensor Network organization

The number and the type of biosensors vary from one patient to another depending on the state of the patient. The most common types of biosensors are EEG "Electroencephalography" to measure the electrical activity produced by the brain, ECG "Electrocardiogram" to record the electrical activity of the heart over time, EMG "Electromyography" to evaluate physiologic properties of muscles, Blood pressure, Glucose monitor, heart rate, Thermometer, $\mathrm{SpO} 2$ "Oxymeter" to measure of oxygen saturation in blood etc...

\subsubsection{Topology}

According to [13-14], Star, Mesh and Spanning Tree based topologies are applicable to BSN. With regards to our BSN architecture, which supports IEEE 802.15.4a UWB compliant sensors, the use of a star topology is the best choice, for the following reasons. First, for a small centralised network of just $4 \mathrm{~m}^{2}$ of scale, a star topology is sufficient. Secondly, the star topology presents several advantages such as (1) simplicity of deployment and management (2) low power consumption of biosensor nodes (3) low latency and less need in terms of bandwidth (only one frequency channel). In fact, there is no need to implement routing protocols in a star-based network, which reduce the complexity of network. Devices or biosensors can only exchange information with the BSN coordinator that might often be main-powered. In 
our case, we admit that the BSN coordinator has less resource constraints than the case of slave nodes. To avoid the "single point of failure" problem in the star topology, we propose that the BSN support the use of a second coordinator. The BSN switches from the first coordinator to the second coordinator only if the first former fails or has a battery level lower than $50 \%$. As illustrated in Fig. 6, in a BSN star topology we distinguish two entities:

1. The BSN Coordinator. It represents the coordinator of the network, which is characterized by its single identifier. In other words, each patient is identified by a unique identifier, in a network of patients. The BSN Coordinator must ensure the following operations:

- Synchronization of the BSN network and with its PAN coordinator,

- GTS management according to the type of applications and the state of patients,

- Duty cycle management within its BSN, according to the density of biosensors per application, the type of application and the state of patients,

- Data Routing of BSN physiological measurements toward PAN coordinator of second network tier,

- Measurements for localization: A BSN coordinator periodically performs measurements of localization in collaboration with the routers of its vicinity,

- Update of allocated frequency channels used inside its own network and the ones used for the routing of BSN physiological measurements inside PAN,

- Priority scheduling to ensure the management of priority per service.

2. Slave Nodes (Biosensors). They must perform physiological measurements and monitoring according to the underlying application (e.g. measurement of the level of glucose in blood for the case of diabetic patient and the report of alarms once the level exceeds the lower or higher limits).

\subsubsection{Operational mode}

We propose the use of the beacon-enabled mode of the IEEE 802.15.4 protocol with GTS (Guaranteed Time Slot) allocation to support real-time applications. Thus, contrarily to Reference [15] that deployed the non beacon-enabled mode for their BSNs based on IEEE 802.15.4a/CSS system, we consider that the beacon-enabled mode is efficient in our model since we can use optional high data rates of 6 or 27 Mbps to shorten transmission delays and to satisfy application requirements without compromising reliability.

In this mode, the BSN coordinator periodically broadcasts, at the mandatory rate of $850 \mathrm{kbps}$, beacon frames containing BSN information in order to synchronize its associated devices and to identify its BSN.

During the superframe duration, two data transfer modes are permitted:

- Transfer from a biosensor to the BSN coordinator: a device willing to transfer physiological information or alarm to the BSN coordinator uses slotted ALOHA with the allocation of guaranteed time slots for the most critical information. The 
BSN coordinator may confirm the successful data reception with an optional acknowledgment message within the same Aloha slot.

- Transfer from the BSN coordinator to a biosensor: when the BSN coordinator has pending data for a given device, it announces this information in beacon frames. The interested device selects a free slot and sends a data request to the BSN coordinator, indicating that it is ready to receive the data. The request is sent using slotted ALOHA. When the BSN coordinator receives the data request message, it selects a free slot and sends data using slotted ALOHA.

The choice of slotted ALOHA is done to avoid the additional access delay due to the collision avoidance phase adopted in CSMA/CA mechanism given that BSNs generally support light and medium traffic loads (very small collision probability).

\subsubsection{Priority Scheduling}

As detailed above, each biosensor must perform in a first step specific physiological measurements and/or monitoring, and in a second step it must send to its BSN coordinator these measurements, and if necessary, it reports alarms.

As shown in the Table 1, according to the characteristics of physiological measurements or type of application services which can be Real-time or Non realtime with High or Low rate, we classify the traffic into four services classes, where class A is the most critical and D the less critical.

Table 1. Service Calssification of Physiological Measurements

\begin{tabular}{|l|c|c|c|c|}
\hline \multicolumn{1}{|c|}{ Type of Service } & Data rate & Latency & \multicolumn{2}{c|}{ Class of Service } \\
\hline ECG & High & Low & A & Real-time high rate \\
\hline EEG, EOG ,EMG & Low & Low & B & Real-time low rate \\
\hline $\begin{array}{l}\text { Heart rate, Blood pressure, Body } \\
\text { temperature, Glucose monitor }\end{array}$ & Low & High & C & Non Real-time low rate \\
\hline Medical image & High & High & D & Non Real-time high rate \\
\hline
\end{tabular}

During data communication period, biosensors transmit its data using the selected data communication channel and with optional data rates of 6 or $27 \mathrm{Mbps}$ in order to reduce communications delays. If a certain node has very urgent and critical data (which requires reduced delays and high degree of reliability), in this case it requests the allocation of one or several GTS time slots based on the traffic characteristics. The allocation can be explicit by requesting a fixed number of time slots as specified in the standard [16], or can be implicit by sending traffic specification to the BSN coordinator, which will allocate slots accordingly as proposed in [17]. Else, the sensor node can transmit its data without sensing the medium directly after a random time slot units.

The GTS allocation is mainly dedicated to the most critical services, so BSN coordinator must allocate such time slots by order of preference according to its resource allocation scheduler. After receiving physiological information according to its class of services, the BSN coordinator must be able to schedule its query in order to facilitate the transmission of the most critical information. With an optimal priority 
scheduling algorithm, we can reduce delays of critical information and satisfy QoS requirements.

\subsection{Second Tier: PAN}

To improve patient's network performance in a dense hospital environment, we propose overlaying the network of BSNs with a second upper level network.

The hexagonal cell represents the PAN or the second network level. As shown in Fig. 4 and Fig. 6, each PAN is represented by a cell of sensors organized in a mesh topology including one PAN coordinator, several mobile BSN coordinators (one active coordinator per BSN) and several routers which relay sensing information toward PAN coordinator.

\subsubsection{Topology}

For a distributed processing, scalability, large coverage, medium complexity, load balancing and energy consumption balancing that we propose mesh topology for the second level of our network model. With such topology, multi-hop routing can enhance significantly the energy consumption and thus maximize network lifespan by balancing load and energy consumption over the entire network.
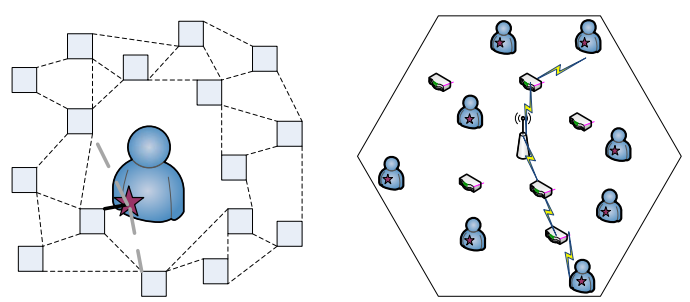

Fig.6 PAN Organization

As shown in the Fig.6 the network is divided into three entities:

1. PAN coordinator, with double interfaces (Wifi/UWB), ensures:

- Synchronization of its network,

- Duty cycle management within its network, according to the density of BSNs, routers and the state of patients,

- Management of time slots per channel allocation inside its cell [18] according to spectrum resource and the priority of resource requests,

- Association and disassociation of BSNs,

- Data Routing: routing of the data of its cell and those of close cells,

- Priority scheduling per patient and service,

- Data security.

2. Routers: They represent fixed UWB sensors mainly acting as relays that ensure:

- Data Routing and management of patients mobility and route update within a cell, 
- Executing some sensing measurements such as humidity, temperature measurements inside the room where they are present.

3. PAN slave nodes: They represent BSNs coordinators.

\subsubsection{Intra-Cell Routing}

For routing inside a cell, we propose using geographic routing algorithm since routers can dispose the location information of each BSN coordinator given that it supports the UWB PHY ranging capability. To deal with energy efficiency/QoS paradox the geographic routing can be optimized by balancing, on the one hand, load and energy consumption all over the network and intelligently allowing routes according to the class of priority of traffic flow, on the other hand.

\subsubsection{Priority Scheduling}

Each BSN represents on reality a patient network, so the state of the patient influences the level of priority of data that it produces. As shown in table 2, according to the state of patient and the characteristics of transmitted information, we classify the traffic into 5 class of services, where class 1 is the most critical and 5 the less critical.

Table 2. Service classification of data inside PAN

\begin{tabular}{|l|l|c|c|}
\hline \multicolumn{2}{|c|}{ Level of critical state } & Latency & Class of service/patient \\
\hline \multicolumn{2}{|l|}{ High critical } & Low & 2 \\
\hline \multirow{2}{*}{ Medium critical } & A, B & Low & 3 \\
\cline { 2 - 3 } & C, D & Medium & 4 \\
\hline \multirow{2}{*}{ Less critical } & A, B & Medium & 5 \\
\cline { 2 - 3 } & C, D & High & 5 \\
\hline \multicolumn{2}{|l}{$\begin{array}{l}\text { Other information (location, } \\
\text { temperature and humidity) }\end{array}$} & Very High & 2 \\
\hline
\end{tabular}

\subsubsection{Operational mode}

In [18], we have proposed a multi-channel MAC protocol for PANs to allow lower latency operation and ensure high throughput without loss on reliability and to maximize network lifespan. For more details, one can refer to multi-channel MAC protocol given in [18].

\subsection{Third Tier: Global WHSN}

For efficient solutions in terms of energy saving, QoS supporting and mobility management inside WHSN that cellular architecture, based on Wifi technology is chosen for the third level to have on global a three-tier hierarchical cellular network. The last tier represents the entire network, where the various entities are found:

- Sink: represents the central station that ensures collection, analysis and treatment of the sensing measurements. We can propose more than one sink according to number of medical data analysis centers.

- Cell coordinator or PAN coordinator: represents UWB/Wifi access points which ensure data collection from patients and inter-cells routing. 
- Intra-cell routers: represent UWB sensors which ensure data routing and some sensing measurements.

- BSN coordinator and its Biosensors members: represent UWB sensors that ensure physiological measurements and medical monitoring.

Fig.7. Global Wireless Hospital Network

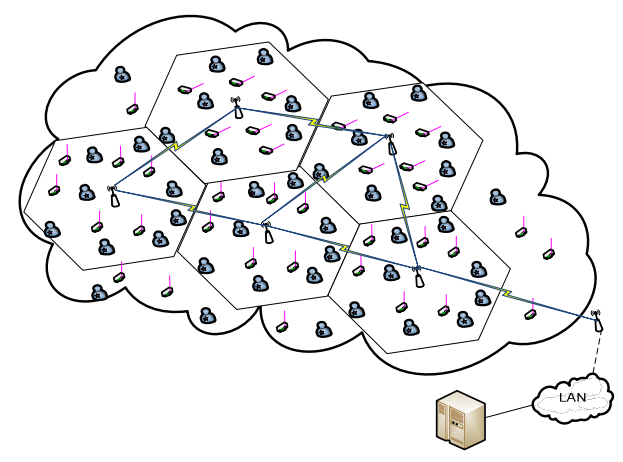

For the inter-cell routing, we propose using mesh multi-hop routing, in order to balance load of entire network. In addition, to deal with energy efficiency/QoS paradox the inter-cell routing can be optimized in order to shorten end to end delays, increase throughput and minimize and balance energy consumption.

\section{Conclusion and Future Work}

In this paper, we have firstly presented a survey of the UWB physical layer that has recently been specified by the IEEE $802.15 .4 \mathrm{a}$ standard, which represents a promising candidate for future cyber-physical systems such as Body Sensor Networks and Home Automation, etc. Then, we have proposed a new WHSN architecture in the form of a UWB/Wifi based three-tiered network to take profit from the interesting features offered by the IEEE 802.15 .4 a UWB physical layer. We believe that our proposed network architecture for healthcare and medical applications in large-scale WHSNs represents a very efficient solution for highly dense networks of patients, thus avoiding congestion and sensors failure caused by energy inefficiency. On the other hand, it ensures the improvement of the network performance in terms of energy efficiency, real-time guarantees and Quality-of-Service (QoS).

Numerous perspectives for designing an optimal WHSN are possible via the proper choice of IEEE 802.15.4a UWB PHY options and the best exploitation of its advantages such as the adaptation of data rate according to the LQI, route selection according to the traffic constraints, CCA mode selection according to application requirements, etc. We are currently working towards the implementation of simulation model of our WHSN architecture using OPNET simulator [19] to evaluate its performance for different network scales (small, medium and large scales) to evaluate the impacts of the number of patients in terms QoS energy consumption, and 
real-time guarantees. In addition, we will propose an efficient channel allocation mechanism for optimizing the use of radio channel in a large-scale WHSN.

\section{References}

1. IEEE 802.15.4a Standard (2007) Part 15.4: IEEE Standard for Information Technology, Amendment to IEEE Std 802.15.4TM-2006, (2007)

2. Tan, A.E.C., Chia, M.Y.W,: Measuring human body impulse response using UWB radar, Electronics Letters, Vol. 41, Iss. 21, (2005) 1193 - 1194

3. Ryckaert, J., Desset, C., Fort, A., Badaroglu, M., De Heyn, V., Wambacq, P., Van der Plas, G., Donnay, S., Van Poucke, B., Gyselinckx, B., : Ultra-Wide Band Transmitter for Wireless Body Area Networks. IEEE Transactions on Circuits and Systems I, Vol.52, No.12, (2005) 2515- 2525

4. Domenicali, D., Di Benedetto, M.-G.,:Performance Analysis for a Body Area Network composed of IEEE 802.15.4a devices. The 4th Workshop on Positioning, Navigation and Communication Hannover, Germany. (2007) 273-276

5. Yang-Sun, L., Jae-Min, K., Sung-Eon, C., Ji-Woong, K., Heau-Jo, K.,:A Study on the Medical Image Transmission Service Based on IEEE 802.15.4a. Springer Berlin / Heidelberg, (2007) 159-167

6. Kottapalli, V-A., Kiremidjian, A-S., Lynch, J-P., Carryer, E., Kenny, T-W., Law, K-H., Lei,Y., :Two-tiered wireless sensor network architecture for structural health monitoring. $10^{\text {th }}$ Annual International Symposium on Smart Structures and Materials, USA (2003)

7. Bin, Z., Chao, H., HaiBin, W., Ruiwen G., Meng, M.Q-H., : A wireless Sensor Network for Pervasive Medical Supervision. IEEE International Conference on Integration Technology, Shenzhen, China (2007) 740-744

8. OTTO, C., Milenkovic, A., Sanders, C., Jovanov, E.,: System Architecture of A Wireless Body Area Sensor Network For Ubiquitous Health Monitoring. Journal of Mobile Multimedia, Vol. 1. No.4 (2006) 307-326

9. Milenkovic, A., OTTO, C., Jovanov, E.,: Wireless Sensor Network for Personal Health Monitoring Issues and an Implementation. Computer Communications. Elsevier (2006)

10. She, H., Lu, Z., Jantsch, A., Zheng, L-R., Zhou, D., : A Network-based System Architecture for Remote Medical Applications. Network Research Workshop (2007)

11. Hongliang, R., Meng, M-Q-H., Xijun, C.,: Physiological Information Acquisition through Wireless Biomedical Sensor Networks. Hong Kong and Macau, China (2005) 483-488

12. Hongliang, R., Meng, M- Q-H., Xijun, C., Haibin, S., Bin, F.,Yawen, C., : System Architecture of Body Area Network and Its Web Services Based Data Publishing. Springer Berlin / Heidelberg. 947-954

13. Espina, J., Falck, T., Mülhens, O.,: Network Topologies, Communication Protocols, and Standards. Spriger book. Body Sensor Networks 145-182

14. http://www.imec.be/wwwinter/mediacenter/en/SR2006/681579.htm

15. Bin, Z., Huan-Bang, L., Ryuji, K., : IEEE Body Area Networks for Medical Applications. IEEE International Symposium on Wireless Communications Systems (2007) 327-331

16. IEEE 802.15.4 Standard Part 15.4: Wireless medium access control (MAC) and physical layer (PHY) specifications for Low-Rate Wireless Personal Area Networks (LR-WPANs), IEEE Standard for Information Technology, Revision of IEEE Std 802.15.4-2003, 2006.

17. Koubâa, A., Alves. M., Tovar. E., : i-GAME: An Implicit GTS Allocation Mechanism in IEEE 802.15.4, In Euromicro Conference on Real-Time Systems (2006)

18. Ben Slimane, J., Song, Y-Q., Frikha, M., Koubâa, A.,:A multi-channel mac protocol for wireless hospital sensor networks, Technical report, 2008, http://hal.inria.fr/inria00322584/fr/.

19. http://www.opnet.com/ 